\title{
EFEITO DE MANEJOS DE SOLO E DE ROTAÇÃO DE CULTURAS DE INVERNO NO RENDIMENTO E DOENÇAS DE TRIGO'
}

\author{
HENRIQUE PEREIRA DOS SANTOS ${ }^{2}$, JULIO CESAR BARRENECHE LHAMBY ${ }^{3}$, \\ ARIANO MORAES PRESTES ${ }^{4}$ e MARCOS ROBERTO DE LIMA ${ }^{5}$
}

\begin{abstract}
RESUMO - O objetivo deste trabalho foi avaliar os efeitos de sistemas de manejo de solo e de rotação de culturas de inverno sobre o rendimento de grãos e sobre doenças radiculares do trigo. Foram comparados quatro sistemas de manejo de solo: plantio direto, cultivo mínimo, preparo convencional do solo com arado de discos mais grade de discos e preparo convencional de solo com arado de aivecas mais grade de discos. Na rotação de culturas, três sistemas foram utilizados: sistema I (trigo/soja), sistema II (trigo/soja e ervilhaca/milho ou sorgo) e sistema III (trigo/soja, aveia-preta ou aveia-branca/soja e ervilhaca/milho ou sorgo). O delineamento experimental foi em blocos ao acaso, com parcelas subdivididas e três repetições. A parcela principal foi constituída pelos sistemas de manejo de solo, e as subparcelas, pelos sistemas de rotação de culturas. O rendimento de grãos de trigo cultivado em plantio direto e em cultivo mínimo foi superior ao cultivado após preparo convencional do solo com arado de discos e com arado de aivecas. A rotação de culturas foi eficiente na redução de doenças do sistema radicular, resultando em aumento do rendimento de grãos de trigo.
\end{abstract}

Termos para indexação: preparo convencional de solo, cultivo mínimo, plantio direto, doenças radiculares.

\section{EFFECT OF SOIL MANAGEMENT AND OF CROP ROTATION SYSTEMS ON WHEAT YIELD AND DISEASES}

\begin{abstract}
The effects of soil management systems and winter crop rotation on wheat yield and root diseases were assessed. No-tillage, minimum tillage, conventional tillage using a disc plow plus disc harrow, and tillage using a moldboard plow plus disc harrow were the soil managements compared. In crop rotation, three systems were used:system I (wheat/soybean), system II (wheat/soybean and common vetch/corn or sorghum), and system III (wheat/soybean, black oats or white oats/soybean, and common vetch/corn or sorghum). A randomized block experimental design, with split-plots and three replicates, was used. The main plots were formed by the soil management systems, while the split-plots consisted of crop rotation systems. Yield of wheat grown under no-tillage and minimum tillage was higher than the yield of wheat grown under conventional soil tillage using either disc plow or moldboard plow. Crop rotation was efficient in reducing root diseases and in increasing wheat yield.

Index terms: conventional tillage, minimum tillage, direct sowing, root diseases.
\end{abstract}

${ }^{1}$ Aceito para publicação em 26 de julho de 2000.

${ }^{2}$ Eng. Agrôn., Dr., Embrapa-Centro Nacional de Pesquisa de Trigo (CNPT), Caixa Postal 451, CEP 99001-970 Passo Fundo, RS. Bolsista do CNPq. E-mail: hpsantos@cnpt.embrapa.br

${ }^{3}$ Eng. Agrôn., Dr., Embrapa-CNPT. E-mail: julio@cnpt.embrapa.br

${ }^{4}$ Eng. Agrôn., Ph.D., Embrapa-CNPT. Bolsista do CNPq. E-mail: ariano@cnpt.embrapa.br

${ }^{5}$ Eng. Agrôn., Coopermarau, Caixa Postal 45, CEP 99150-000 Marau, RS.

\section{INTRODUÇÃO}

Sistemas de manejo de solo, compatíveis com características do clima, da planta e do solo na Região Sul do Brasil, são imprescindíveis para interromper o processo de degradação dos solos e para promover sua recuperação, mantendo a lavoura economicamente integrada no sistema de produção agrícola (Reunião, 1998). Nesse contexto, o plantio di- 
reto constitui o manejo de solo com maior potencial para atender a esses objetivos.

O sistema plantio direto, por sua vez, está diretamente associado à cobertura morta para proteger o solo. A manutenção dessa cobertura morta só tornarse-á viável por meio de sistemas adequados de rotação de culturas (Santos et al., 1993).

Ruedell (1995), na região de Cruz Alta, RS, trabalhando com sistemas de manejo do solo, observou, entre as culturas, que o trigo foi a espécie que demonstrou menor resposta a essa prática agrícola. Entretanto, no período estudado e na maioria dos anos, trigo cultivado sob plantio direto apresentou rendimento de grãos mais elevado do que sob preparo convencional do solo.

A rotação de culturas permite produzir e estabilizar o rendimento de grãos pela diversificação de espécies (Ruedell, 1995; Santos et al., 1996, 1998). Isso ocorre em virtude da completa decomposição microbiana de resíduos vegetais e, conseqüentemente, eliminação do inóculo da área de cultivo (Reis, 1991). A mineralização de resíduos vegetais, para completa eliminação de doenças do sistema radicular (podridão-comum e mal-de-pé), pode levar de 12 a 16 meses (Reis \& Santos, 1993).

Ledinghan (1961), no Canadá, observou que a monocultura de trigo apresentou valores mais elevados de severidade de podridão-comum, em comparação a um, dois, três e cinco invernos sem esse cereal. Slope et al. (1973), na Inglaterra, verificaram diferentes valores de severidade do mal-do-pé entre monocultura e dois invernos sem trigo. Santos et al. (1996), no Estado do Paraná, relataram menor rendimento de grãos e valores mais elevados de severidade do mal-do-pé e da podridão-comum, na monocultura de trigo, sob plantio direto, quando comparados a um, dois ou três invernos sem trigo.

Slope et al. (1973), na Inglaterra, trabalhando com sistemas de rotação, observaram que a monocultura de trigo apresentou menor rendimento de grãos, em relação a dois invernos sem trigo. Sturz \& Bernier (1989), no Canadá, verificaram menor rendimento de grãos na monocultura de trigo, em comparação a um inverno sem esse cereal, alternado com canola ou com linho.

A cultura de trigo, para completar seu ciclo, requer aproximadamente $322 \mathrm{~mm}$ de precipitação plu- vial (Matzenauer, 1992). A precipitação normal (822 mm), na região de Passo Fundo, tem sido acima desse valor (Brasil, 1992). Além disso, quando houve excesso de precipitação pluvial, os efeitos positivos da rotação de culturas foram mais bem demonstrados pela estabilidade do rendimento de grãos e pelo controle de doenças do sistema radicular de trigo (Santos et al., 1996, 1998).

O presente trabalho teve por objetivo avaliar o efeito de sistemas de manejo de solo e de rotação de culturas no rendimento de grãos de trigo.

\section{MATERIAL E MÉTODOS}

O ensaio foi conduzido na Embrapa-Centro Nacional de Pesquisa de Trigo (CNPT), no Município de Passo Fundo, RS, no período de 1986 a 1997, em solo classificado como Latossolo Vermelho distrófico típico (Brasil, 1973). A área experimental vinha sendo cultivada com trigo, no inverno, e com soja, no verão.

Os tratamentos foram constituídos por quatro sistemas de manejo de solo - 1) plantio direto, 2) preparo de solo com implemento de cultivo mínimo JAN; 3) preparo convencional do solo com arado de discos mais grade de discos; 4) preparo convencional do solo com arado de aivecas mais grade de discos - e por três sistemas de rotação de culturas: sistema I (trigo/soja), sistema II [trigo/soja e ervilhaca/milho (de 1986 a 1993) ou sorgo (de 1994 a 1996) ou milho (em 1997)] e sistema III [trigo/soja, aveiapreta (de 1986 a 1989) ou aveia-branca (de 1990 a 1997)/ soja e ervilhaca/milho (de 1986 a 1993) ou sorgo (de 1994 a 1996) ou milho (em 1997)]. As cultivares de trigo usadas foram BR-14, em 1988 e em 1989, BR-23, de 1990 a 1993, e Embrapa 16, de 1994 a 1997. Em 1990, foi alterada a sequiencia do sistema III, ficando trigo/soja, ervilhaca/milho e aveia-branca/soja.

Em novembro de 1985, antes da instalação do experimento, foram realizadas a descompactação e a correção da acidez com calcário, de acordo com os resultados da análise de solo da área experimental. As amostragens de solo, para determinação dos níveis de nutrientes e do teor de matéria orgânica, foram realizadas anualmente em todas parcelas, após a colheita das culturas de verão com a profundidade de $0-20 \mathrm{~cm}$ (Tabela 1).

A semeadura, o controle de plantas daninhas e os tratamentos fitossanitários foram realizados conforme recomendação para cada cultura, e a colheita de grãos foi efetuada com colhedora especial para parcelas experimentais. O rendimento de grãos de trigo foi determinado a partir 
da colheita de toda parcela, ajustando-se o rendimento para umidade de $13 \%$.

A avaliação do grau de severidade de doenças do sistema radicular de trigo (mal-do-pé, causado por Gaeumannomyces graminis var. tritici, e podridão-comum, causada por Bipolaris sorokiniana) foram efetuadas de acordo com o método empregado por Reis et al. (1985). Os dados originais foram transformados em arco seno $\sqrt{\mathrm{x}}$ para análise da severidade de doenças do sistema radicular. A severidade de doenças do sistema radicular e o rendimento de grãos de trigo foram avaliados a partir de 1988, ano em que se completou o primeiro ciclo de rotação, possibilitando observar o sistema de rotação com dois invernos sem trigo.

Foram obtidos os dados de precipitação pluvial observados durante o período do experimento, no posto meteorológico-padrão, localizado na Embrapa-Centro Nacional de Pesquisa de Trigo.

Foi usado delineamento experimental de blocos ao acaso, com parcelas subdivididas e três repetições. A parcela principal foi constituída pelos sistemas de manejo de solo, e as subparcelas, pelos sistemas de rotação de culturas. A parcela principal media $360 \mathrm{~m}^{2}$ (4 m de largura por $90 \mathrm{~m}$ de comprimento), e a subparcela, $40 \mathrm{~m}^{2}$ (4 $\mathrm{m}$ de largura por $10 \mathrm{~m}$ de comprimento). Foi efetuada análise de variância do rendimento de grãos e da severidade das doenças do sistema radicular de trigo (dentro de cada ano e na média conjunta dos anos de 1988 a 1997). Considerou-se o efeito tratamento (diferentes sistemas de manejos de solo e sistemas de rotação de culturas) como fixo, e o efeito ano, como aleatório. As médias foram comparadas entre si, pelo teste de Duncan, a 5\% de probabilidade.

\section{RESULTADOS E DISCUSSÃO}

A análise conjunta dos resultados para severidade de doenças do sistema radicular e para rendimen- to de grãos de trigo (1988 a 1997) mostrou significância com relação ao efeito anos, aos sistemas de manejo de solo, à rotação de culturas e à interação ano x rotação de culturas. Houve ainda efeito significativo do rendimento de grãos para a interação ano x manejo de solo. O efeito de ano indica que essas variáveis foram afetadas pela variação climática. Não houve diferenças significativas na severidade das doenças do sistema radicular e no rendimento de grãos de trigo com relação às interações sistema de manejo de solo $\mathrm{x}$ rotação de culturas, e ano x sistemas de manejo de solo x rotação de culturas. Resultados similares relativos a ano, sistemas de manejo do solo, rotação de culturas, interação de sistemas de manejo de solo x rotação de culturas e interação ano $\mathrm{x}$ sistemas de manejo de solo $\mathrm{x}$ rotação de culturas foram relatados por Langdale et al. (1990). Nesse caso, houve aumento de rendimento de grãos, em decorrência da rotação de culturas, principalmente nas espécies de verão.

A severidade de doenças do sistema radicular de trigo (mal-do-pé e podridão-comum), em 1988, 1989, 1992 e 1995, e na média dos anos, diferiu significativamente entre os sistemas de manejo de solo (Tabela 2). Os valores mais elevados manifestaram-se no sistema plantio direto, no preparo convencional de solo com arado de discos e no cultivo mínimo, em relação ao preparo convencional do solo com arado de aivecas. Essas diferenças podem ser atribuídas ao fato de o arado de aivecas enterrar os resíduos culturais de trigo a uma maior profundidade do que nos demais manejos do solo, tornando a decomposição mais rápida, e assim, eliminando a fonte de substrato para multiplicação de patógenos causadores dessas doenças.

TABELA 1. Valores médios de pH, alumínio, cálcio + magnésio, matéria orgânica, fósforo e potássio, determinados em amostras $(0-20 \mathrm{~cm})$ coletadas após a colheita das culturas de verão, nos diferentes anos.

\begin{tabular}{|c|c|c|c|c|c|c|c|c|c|c|}
\hline Características do solo $^{1}$ & 1988 & 1989 & 1990 & 1991 & 1992 & 1993 & 1994 & 1995 & 1996 & 1997 \\
\hline pH em água (1:1) & 5,3 & 5,4 & 5,9 & 5,5 & 5,2 & 5,5 & 5,4 & 5,1 & 5,3 & 5,4 \\
\hline $\mathrm{Al}$ trocável $\left(\mathrm{mmol}_{\mathrm{c}} / \mathrm{dm}^{3}\right)$ & 4,4 & 3,1 & 1,3 & 2,8 & 5,8 & 4,0 & 3,6 & 7,1 & 7,0 & 6,7 \\
\hline $\mathrm{Ca}+\mathrm{Mg}$ trocáveis $\left(\mathrm{mmol}_{\mathrm{c}} / \mathrm{dm}^{3}\right)$ & 81,5 & 93,6 & 82,2 & 83,7 & 74,4 & 72,9 & 79,3 & 57,8 & 59,9 & 61,9 \\
\hline Matéria orgânica $(\mathrm{g} / \mathrm{kg})$ & 44 & 42 & 39 & 47 & 25 & 27 & 22 & 25 & 30 & 25 \\
\hline P extraível (mg/kg) & 21,0 & 21,7 & 13,3 & 10,5 & 15,9 & 13,2 & 35,2 & 12,8 & 14,8 & 12,3 \\
\hline K trocável (mg/kg) & 135 & 151 & 143 & 143 & 120 & 131 & 122 & 99 & 107 & 106 \\
\hline
\end{tabular}

${ }^{1}$ Média de todas as parcelas. 
Na maioria dos anos e na média dos anos, houve diferenças significativas na severidade de doenças do sistema radicular de trigo, decorrentes dos sistemas de rotação de culturas (Tabela 3 ). Os valores mais elevados de severidade do mal-do-pé e de podridão-comum de raízes ocorreram na monocultura de trigo, em comparação com um inverno e com dois invernos sem essa gramínea, demonstrando assim o efeito positivo da rotação com espécies não-suscetíveis.

Ledinghan (1961), em trabalho conduzido no Canadá, relatou valores mais elevados de severidade da podridão-comum de raízes de trigo em monocultura (68\%), em relação a um (64\%), a dois (37\%), a três $(34 \%)$ e a cinco invernos (14\%) sem esse cereal.

TABELA 2. Efeito de sistemas de manejo do solo na severidade de doenças do sistema radicular e no rendimento de grãos de trigo, em 1988 e em 1989, cultivar BR-14, de 1990 a 1993 , cultivar BR-23, e de 1994 a 1997, cultivar Embrapa 16. Passo Fundo, RS1.

\begin{tabular}{|c|c|c|c|c|c|}
\hline \multirow[t]{2}{*}{ Ano } & \multicolumn{4}{|c|}{ Manejo de solo ${ }^{2}$} & \multirow[t]{2}{*}{ Média } \\
\hline & PD & PCD & PCA & PM & \\
\hline & \multicolumn{5}{|c|}{ Severidade de doenças do sistema radicular de trigo (\%) } \\
\hline 1988 & $30 \mathrm{Aa}$ & $32 \mathrm{Aa}$ & 22Bab & $22 \mathrm{Bb}$ & $27 \mathrm{~b}$ \\
\hline 1989 & $19 \mathrm{ABc}$ & 28Aabc & $16 \mathrm{Bbc}$ & $23 \mathrm{Ab}$ & $21 \mathrm{~cd}$ \\
\hline 1990 & $23 \mathrm{Abc}$ & 25Aabcd & $21 \mathrm{Aab}$ & $24 \mathrm{Ab}$ & $23 \mathrm{bcd}$ \\
\hline 1991 & $16 \mathrm{Ac}$ & $14 \mathrm{Ae}$ & $15 \mathrm{Abc}$ & $13 \mathrm{Ac}$ & $15 \mathrm{f}$ \\
\hline 1992 & $18 \mathrm{Bc}$ & $30 \mathrm{Aab}$ & $28 \mathrm{Aa}$ & $27 \mathrm{Aab}$ & $26 b$ \\
\hline 1993 & $34 \mathrm{Aa}$ & $33 \mathrm{Aa}$ & $27 \mathrm{Aa}$ & $34 \mathrm{Aa}$ & $32 a$ \\
\hline 1994 & $16 \mathrm{Ac}$ & 21Acde & $12 \mathrm{Ac}$ & $19 \mathrm{Ab}$ & $17 \mathrm{e}$ \\
\hline 1995 & $24 \mathrm{Abc}$ & 19Ade & $15 \mathrm{Bbc}$ & $25 \mathrm{Aab}$ & $21 \mathrm{~cd}$ \\
\hline 1996 & $23 \mathrm{Abc}$ & 22Acd & 19Aabc & $23 \mathrm{Ab}$ & $22 \mathrm{~cd}$ \\
\hline 1997 & $24 \mathrm{Ab}$ & $24 \mathrm{Abcd}$ & $23 \mathrm{Aa}$ & $27 \mathrm{Aa}$ & $25 \mathrm{bc}$ \\
\hline \multirow[t]{2}{*}{ Média } & $23 \mathrm{~A}$ & $25 \mathrm{~A}$ & $20 \mathrm{~B}$ & $24 \mathrm{~A}$ & 23 \\
\hline & \multicolumn{5}{|c|}{ Rendimento de grãos de trigo (kg/ha) } \\
\hline 1988 & $2.183 \mathrm{Ad}$ & $1.905 \mathrm{Ad}$ & $1.906 \mathrm{Ac}$ & 2.194Ad & $2.047 \mathrm{e}$ \\
\hline 1989 & $2.909 \mathrm{Ac}$ & $3.013 \mathrm{Ab}$ & $2.871 \mathrm{Ab}$ & $2.959 \mathrm{Ac}$ & $2.938 b c$ \\
\hline 1990 & $3.167 \mathrm{Ab}$ & $2.429 \mathrm{Cc}$ & $2.789 \mathrm{Bb}$ & $3.082 \mathrm{Ab}$ & $2.866 \mathrm{c}$ \\
\hline 1991 & $2.968 \mathrm{Ac}$ & $2.971 \mathrm{Ab}$ & $2.886 \mathrm{Ab}$ & $2.957 \mathrm{Ac}$ & $2.946 \mathrm{~b}$ \\
\hline 1992 & $4.151 \mathrm{Aa}$ & $4.031 \mathrm{Aa}$ & $3.820 \mathrm{Ba}$ & $3.942 \mathrm{Aba}$ & $3.986 a$ \\
\hline 1993 & $2.318 \mathrm{Ad}$ & $1.665 \mathrm{Be}$ & $1.379 \mathrm{Be}$ & $2.243 \mathrm{Ad}$ & $1.901 \mathrm{e}$ \\
\hline 1994 & $3.407 \mathrm{Ab}$ & $2.776 \mathrm{Bb}$ & $2.853 \mathrm{Bb}$ & $3.322 \mathrm{Ab}$ & $3.090 \mathrm{~b}$ \\
\hline 1995 & $2.385 \mathrm{Ad}$ & $2.181 \mathrm{Ac}$ & $2.101 \mathrm{Ac}$ & $2.244 \mathrm{Ad}$ & $2.228 \mathrm{~d}$ \\
\hline 1996 & $1.868 \mathrm{Ae}$ & 1.874Ade & 1.810Acd & $1.953 \mathrm{Ad}$ & $1.876 \mathrm{f}$ \\
\hline 1997 & $1.910 \mathrm{Ae}$ & $1.790 \mathrm{Ae}$ & $1.702 \mathrm{Ad}$ & $1.714 \mathrm{Ae}$ & $1.779 \mathrm{f}$ \\
\hline Média & $2.727 \mathrm{~A}$ & $2.463 \mathrm{~B}$ & $2.412 B$ & $2.661 \mathrm{~A}$ & 2.566 \\
\hline
\end{tabular}

1 Médias seguidas da mesma letra, minúscula na vertical e maiúscula na horizontal, não apresentam diferenças significativas, a 5\% de probabilidade, pelo teste de Duncan.

2 PD: plantio direto; PCD: preparo convencional de solo com arado de discos; PCA: preparo convencional de solo com arado de aivecas; PM: cultivo mínimo.
Esses resultados mostram decréscimo da doença proporcionalmente aos anos de rotação de culturas. Slope et al. (1973), na Inglaterra, observaram diferentes valores de severidade do mal-do-pé entre monocultura $(35 \%)$ e dois anos de rotação de culturas não-suscetíveis (3\%). Santos et al. (1996), no Estado do Paraná, sob plantio direto, verificaram valores mais elevados de severidade de podridões de raízes de trigo em monocultura (39\%) do que em trigo cultivado em rotação de um (12\%), dois (9\%) e três invernos (11\%). Santos et al. (1998), no Estado do Rio Grande do Sul, sob preparo convencional do solo, no inverno, e sob plantio direto, no verão, relataram os seguintes porcentuais de severidade do mal-dopé e da podridão-comum: monocultura de trigo (50\%), um (13\%), dois (10\%), três invernos (10\%), dois invernos sem, e dois com trigo ( $13 \%$ e $16 \%$ ), e três invernos sem, e dois com trigo (12\% e 19\%).

A mais elevada severidade de doenças do sistema radicular do trigo, em todos os sistemas de manejo ou de rotações de culturas, foi verificada no ano de 1993 (32\%) (Tabelas 2 e 3). Deve ser levado em consideração que os valores obtidos com relação a severidade de doenças do sistema radicular do trigo, ao longo desse anos, mantiveram-se em limiar relativamente baixo, mesmo na monocultura de trigo (33\%).

O rendimento de grãos de trigo, nos anos de 1990, 1992, 1993 e 1994, e na média dos anos (Tabela 2), apresentou diferenças significativas entre os sistemas de manejo de solo. O rendimento mais elevado ocorreu no sistema plantio direto e no cultivo mínimo, em comparação com os de preparo convencional do solo com arado de discos ou com arado de aivecas. Essas diferenças podem ser atribuídas a deficiências hídricas em alguma fase crítica de desenvolvimento da cultura.

De maneira geral, as espécies possuem um estádio de desenvolvimento no qual a deficiência hídrica causa maior redução no rendimento de grãos. Segundo Rodrigues et al. (1998), em trigo, isso ocorre do estádio de folha-bandeira ao estádio de antese, que, nas condições da região sul-brasileira, na maioria das vezes, manifesta-se no final de agosto. A quantidade de precipitação requerida para o trigo completar seu ciclo é de aproximadamente $322 \mathrm{~mm}$ (Matzenauer, 1992). Entretanto, na região de Passo Fundo, RS, a precipitação pluvial normal é de $822 \mathrm{~mm}$ (Brasil, 
1992). No decorrer dos anos de condução do presente trabalho, a precipitação pluvial foi mal distribuída, principalmente no mês de agosto de 1988 (28 mm), 1990 (38 mm), 1993 (15 mm) e 1994 $(46 \mathrm{~mm})$. Isso pode explicar, em parte, a diferença em rendimento de grãos entre sistemas conservacionistas do solo e sistemas convencionais de preparo do solo, uma vez que os primeiros apresentam condições de armazenar mais umidade para ser usada nos períodos mais críticos do desenvolvimento de trigo.

Em pesquisa realizada por Porto (1980), em Passo Fundo, RS, com sistemas de manejo de solo, observou-se que o trigo estabelecido sob sistema plantio direto produziu mais do que sob preparo convencional de solo. Em trabalho conduzido por Ruedell
(1995), em Cruz Alta, RS, com sistemas de manejo do solo, o trigo foi a espécie que sofreu menor influência dessa prática agrícola. Dos nove anos estudados, em oito anos o sistema plantio direto apresentou rendimento de grãos mais elevado do que preparo convencional do solo.

Na maioria dos anos e na média dos anos, o rendimento de grãos de trigo cultivado nos três sistemas de rotação de culturas diferiu significativamente (Tabela 3). O sistema de rotação de culturas com dois invernos sem trigo mostrou rendimento médio de grãos mais elevado do que sob monocultura e sob um inverno sem trigo. O menor rendimento de grãos de trigo ocorreu na monocultura desse cereal.

O trigo após milho ou sorgo (com um inverno de rotação) apresentou rendimento de grãos intermedi-

TABELA 3. Efeito de sistemas de rotação de culturas na severidade de doenças do sistema radicular e no rendimento de grãos de trigo, em 1988 e em 1989, cultivar BR-14, de 1990 a 1993, cultivar BR-23, e de 1994 a 1997, cultivar Embrapa 16. Passo Fundo, RS ${ }^{1}$.

\begin{tabular}{|c|c|c|c|c|}
\hline \multirow[t]{2}{*}{ Ano } & \multicolumn{3}{|c|}{ Sistema de rotação } & \multirow[t]{2}{*}{ Média } \\
\hline & Monocultura & Um inverno sem trigo & Dois invernos sem trigo & \\
\hline \multicolumn{5}{|c|}{ Severidade de doenças do sistema radicular de trigo (\%) } \\
\hline 1988 & 37Aab & $22 \mathrm{Bb}$ & $21 \mathrm{Bb}$ & $27 \mathrm{~b}$ \\
\hline 1989 & $25 \mathrm{Ac}$ & $18 \mathrm{Abc}$ & $21 \mathrm{Ab}$ & $21 \mathrm{~cd}$ \\
\hline 1990 & $28 \mathrm{Ac}$ & $21 \mathrm{Ab}$ & $20 \mathrm{Bb}$ & $23 \mathrm{bcd}$ \\
\hline 1991 & $24 \mathrm{Ac}$ & $9 \mathrm{Be}$ & $10 \mathrm{Bc}$ & $14 \mathrm{f}$ \\
\hline 1992 & 37Aab & $20 \mathrm{Bb}$ & $20 \mathrm{Bb}$ & $26 \mathrm{~b}$ \\
\hline 1993 & 36Aab & $31 \mathrm{Aa}$ & 29Aа & $32 \mathrm{a}$ \\
\hline 1994 & $31 \mathrm{Abc}$ & 11Bde & $8 \mathrm{Bd}$ & $17 \mathrm{e}$ \\
\hline 1995 & $32 \mathrm{Ab}$ & 14Bbcde & $17 \mathrm{Bb}$ & $21 \mathrm{~cd}$ \\
\hline 1996 & $43 \mathrm{Aa}$ & 13Bcde & $10 \mathrm{Bcd}$ & $22 \mathrm{~cd}$ \\
\hline 1997 & 38Aa & 17Bbcd & $19 \mathrm{Bb}$ & $25 \mathrm{bc}$ \\
\hline Média & $33 \mathrm{~A}$ & 18B & $17 \mathrm{~B}$ & 23 \\
\hline \multicolumn{5}{|c|}{ Rendimento de grãos (kg/ha) } \\
\hline 1988 & $2.006 \mathrm{Ac}$ & $2.088 \mathrm{Ac}$ & $2.048 \mathrm{Ag}$ & $2.047 \mathrm{e}$ \\
\hline 1989 & $2.970 \mathrm{Aa}$ & $2.919 \mathrm{Ab}$ & $2.925 \mathrm{Ad}$ & $2.938 \mathrm{bc}$ \\
\hline 1990 & $2.527 \mathrm{Bb}$ & $2.968 \mathrm{Ab}$ & $3.105 \mathrm{Ac}$ & $2.866 \mathrm{c}$ \\
\hline 1991 & $2.532 \mathrm{Bb}$ & $3.089 \mathrm{Ab}$ & $3.216 \mathrm{Ab}$ & $2.946 b$ \\
\hline 1992 & $3.158 \mathrm{Ca}$ & $4.076 \mathrm{Ba}$ & $4.724 \mathrm{Aa}$ & $3.986 \mathrm{a}$ \\
\hline 1993 & $1.012 \mathrm{Be}$ & $2.248 \mathrm{Ac}$ & $2.443 \mathrm{Ae}$ & $1.901 \mathrm{e}$ \\
\hline 1994 & $2.663 \mathrm{Bb}$ & $3.210 \mathrm{Ab}$ & $3.395 \mathrm{Ab}$ & $3.089 \mathrm{~b}$ \\
\hline 1995 & $1.988 \mathrm{Bc}$ & $2.059 \mathrm{Bcd}$ & 2.637Ae & $2.228 \mathrm{~d}$ \\
\hline 1996 & $1.526 \mathrm{Cd}$ & $1.829 \mathrm{Bd}$ & $2.274 \mathrm{Af}$ & $1.876 \mathrm{f}$ \\
\hline 1997 & $1.648 \mathrm{Bd}$ & $1.714 \mathrm{Be}$ & $1.975 \mathrm{Ag}$ & $1.779 \mathrm{f}$ \\
\hline Média & $2.203 \mathrm{C}$ & $2.620 \mathrm{~B}$ & $2.874 \mathrm{~A}$ & 2.566 \\
\hline
\end{tabular}


ário entre a monocultura desse cereal e rotação de dois invernos (Tabela 3). Nos anos de 1995 e 1996, observou-se que o trigo após sorgo mostrou, ao longo de seu ciclo, menor estatura de plantas, e folhas com coloração verde menos intensa, em comparação a trigo após soja (dois invernos de rotação e monocultura). Como nesse sistema havia um inverno de rotação, era esperado que o rendimento de grãos fosse semelhante estatisticamente ao de trigo com dois invernos de rotação, conforme observado em estudos com sistemas de rotação para essa espécie (Santos et al., 1996, 1998).

Triñanes Schaffner \& Uriarte Bregante (1984) avaliaram os efeitos residuais de girassol, de milho, de soja e de sorgo sobre o crescimento e sobre o rendimento de grãos de trigo, no Uruguai. Todos os resíduos vegetais apresentaram efeitos alelopáticos sobre o rendimento de grãos de trigo, mas o resíduo de sorgo foi o que mais afetou negativamente a cultura do trigo.

Vários estudos mostram menor rendimento de grãos de trigo em monocultura. Slope et al. (1973), na Inglaterra, verificaram que a monocultura de trigo apresentou menor rendimento de grãos $(4.530 \mathrm{~kg} / \mathrm{ha})$ do que dois invernos $(5.460 \mathrm{~kg} / \mathrm{ha})$ sem trigo, e Sturz \& Bernier (1989), no Canadá, observaram que a monocultura de trigo $(3.179 \mathrm{~kg} / \mathrm{ha})$ rendeu menos que um inverno sem esse cereal alternado com canola (4.203 kg/ha) ou com linho (4.076 kg/ha). Santos et al. (1996), em experimentos sob plantio direto, no Estado do Paraná, relataram menor rendimento de grãos na monocultura de trigo $(3.014 \mathrm{~kg} / \mathrm{ha})$ do que nos sistemas de rotação com um $(3.355 \mathrm{~kg} / \mathrm{ha})$, dois $(3.494 \mathrm{~kg} / \mathrm{ha})$ e três invernos $(3.362 \mathrm{~kg} / \mathrm{ha})$ sem trigo. No Estado do Rio Grande do Sul, em áreas sob preparo convencional de solo, no inverno, e sob plantio direto, no verão, Santos et al. (1998) também verificaram menor rendimento de grãos na monocultura de trigo $(2.238 \mathrm{~kg} / \mathrm{ha})$ do que um $(3.502 \mathrm{~kg} / \mathrm{ha})$, dois (3.403 kg/ha), três invernos (3.629 kg/ha), dois invernos sem e dois com trigo $(3.476$ e $3.290 \mathrm{~kg} / \mathrm{ha})$ e três invernos sem e dois com trigo ( 3.557 e $3.528 \mathrm{~kg} / \mathrm{ha}$ ).

Rendimento de grãos de trigo mais elevado em todos os sistemas de manejo de solo e de rotação de culturas foi observado no ano de $1992(3.986 \mathrm{~kg} / \mathrm{ha})$ (Tabelas 2 e 3 ), enquanto menor rendimento de grãos ocorreu nos anos de 1996 (1.876 kg/ha) e 1997 (1.779 kg/ha).
O melhor rendimento de grãos de trigo ocorreu nos sistemas de manejo de solo (cultivo mínimo e plantio direto), em comparação com os sistemas de preparo convencional de solo (com arado de discos ou com arado de aivecas).

\section{CONCLUSÕES}

1. O uso da rotação de culturas permite a redução de, aproximadamente, $50 \%$ da severidade de doenças de raízes de trigo.

2. A rotação de culturas aumenta o retorno econômico da lavoura, pelo aumento e estabilidade do rendimento de grãos, oferecendo alternativas de diversificação de culturas e conseqüentemente diminuindo o risco de insucesso do agricultor.

\section{REFERÊNCIAS}

BRASIL. Ministério da Agricultura. Departamento Nacional de Pesquisa Agropecuária. Divisão de Pesquisa Pedológica. Levantamento de reconhecimento dos solos do Estado do Rio Grande do Sul. Recife, 1973. 431p. (Boletim Técnico, 30).

BRASIL. Ministério da Agricultura e da Reforma Agrária. Departamento Nacional de Meteorologia. Normas climatológicas (1961-1990). Brasília, 1992. $84 \mathrm{p}$.

LANGDALE, G.W.; WILSON JUNIOR, R.L.; BRUCE, R.R. Cropping frequencies to sustain long-term conservation tillage systems. Soil Science Society of America. Journal, Madison, v.54, n.1, p.193-198, 1990.

LEDINGHAN, R.J. Crop rotations and common root rot in wheat. Canadian Journal of Plant Science, Ottawa, v.41, n.3, p.479-486, 1961.

MATZENAUER, R. Evapotranspiração de plantas cultivadas e coeficientes de cultura. In: BERGAMASCHI, H.; BERLARTO, M.A.; MATZENAUER, R.; FONTANA, D.C.; CUNHA, G.R.; SANTOS, M.L.V. dos; FARIAS, J.R.B.; BARNI, N.A. (Coord.). Agrometeorologia aplicada à irrigação. Porto Alegre : UFRGS, 1992. p.33-47.

PORTO, V.H. da F. Análise econométrica de dados experimentais sobre um sistema de produção trigo- 
soja, para a cultura de trigo. Piracicaba : ESALQ, 1980. 109p. Dissertação de Mestrado.

REIS, E.M. Potencialidade de controle de doenças de trigo e de cevada por rotação de culturas. In: REUNIÃO BRASILEIRA SOBRE CONTROLE BIOLÓGICO DE DOENÇAS DE PLANTAS, 4., 1991, Campinas. Anais. Campinas : Embrapa-CNPDA, 1991. p.78-99.

REIS; E.M.; SANTOS, H.P. dos. Interações entre doenças de cereais de inverno e sistema plantio direto. In: EMBRAPA. Centro Nacional de Pesquisa de Trigo (Passo Fundo, RS). Plantio direto no Brasil. Passo Fundo : Embrapa-CNPT/FUNDACEP FECOTRIGO/Fundação ABC/Aldeia Norte, 1993. p.105-110.

REIS, E.M.; SANTOS, H.P. dos; PEREIRA, L.R. Rotação de culturas. IV. Efeito sobre o mosaico e doenças radiculares do trigo em 1983. Fitopatologia Brasileira, Brasília, v.10, n.3, p.637-642, 1985.

REUNIÃO DA COMISSÃO SUL-BRASILEIRA DE PESQUISA DE TRIGO, 30., 1998, Chapecó. Recomendações. Chapecó : Comissão Sul-Brasileira de Pesquisa de Trigo, 1998. 82p.

RODRIGUES, O.; LHAMBY, J.C.B.; DIDONET, A.D.; MARCHESE, J.A.; SCIPIONI, C. Efeito da deficiência hídrica na produção de trigo. Pesquisa Agropecuária Brasileira, Brasília, v.33, n.6, p.839-846, jun. 1998.

RUEDELL, J. Plantio direto na região de Cruz Alta. Cruz Alta : FUNDACEP/FECOTRIGO, 1995. 134p.
SANTOS, H.P. dos; LHAMBY, J.C.B.; PRESTES, A.M.; REIS, E.M. Características agronômicas e controle de doenças radiculares de trigo, em rotação com outras culturas de inverno. Pesquisa Agropecuária Brasileira, Brasília, v.33, n.3, p.277-288, mar. 1998.

SANTOS, H.P. dos; REIS, E.M.; DERPSCH, R. Rotação de culturas. In: EMBRAPA. Centro Nacional de Pesquisa de Trigo (Passo Fundo, RS). Plantio direto no Brasil. Passo Fundo : Embrapa-CNPT/ FUNDACEP FECOTRIGO/Fundação ABC/Aldeia Norte, 1993. p.85-103.

SANTOS, H.P. dos; REIS, E.M.; LHAMBY, J.C.B.; WOBETO, C. Efeito da rotação de culturas sobre o trigo, em sistema plantio direto, em Guarapuava, PR. Pesquisa Agropecuária Brasileira, Brasília, v.31, n.4, p.259-267, abr. 1996.

SLOPE, D.B.; ETHERIDGE, J.; WILLIAMS, R.J.B. Grain yield and incidence of take-all and eyespot in winter wheat grown in different crop sequences at Saxmundham. Rothamsted Experimental Station Report for 1972, Harpenden, pt. 2, p.160-167, 1973.

STURZ, A.V.; BERNIER, C.C. Influence of crop rotations on winter wheat growth and yield in relation to the dynamics of pathogenic crown and root rot fungal complexes. Canadian Journal of Plant Pathology, Guelph, v.11, n.1, p.114-121, 1989.

TRIÑANES SCHAFFNER, E.; URIARTE BREGANTE, $C$. Efecto residual de rastrojos de girasol, maíz, soja y sorgo en el crecimiento y producción de trigo. Montevideo : Universidad de la RepublicaFacultad de Agronomía, 1984. 196p. 